\title{
Criminologie
}

\section{La peur du crime : mythes et réalités}

\section{Christiane Louis-Guérin}

Volume 16, numéro 1, 1983

La peur du crime

URI : https://id.erudit.org/iderudit/017172ar

DOI : https://doi.org/10.7202/017172ar

Aller au sommaire du numéro

Éditeur(s)

Les Presses de l'Université de Montréal

ISSN

0316-0041 (imprimé)

1492-1367 (numérique)

Découvrir la revue

Citer cet article

Louis-Guérin, C. (1983). La peur du crime : mythes et réalités. Criminologie, 16(1), 69-83. https://doi.org/10.7202/017172ar d'utilisation que vous pouvez consulter en ligne.

https://apropos.erudit.org/fr/usagers/politique-dutilisation/ 


\section{LA PEUR DU CRIME : MYTHES ET RÉALITÉS \\ Christiane Louis-Guérin*}

Mise en scène quotidiennement par les mass-media, la violence semble nous cerner de toutes parts, les agressions se multiplier. De leur côté, les experts tiennent un discours alarmiste : "Longtemps tenue en marge, la violence s'est installée au cœur de la Cité. Pas encore maîtresse, mais ce temps peut venir" (p. 31, Réponses à violence, 1977) ; donnent des raisons pour justifier la nécessité d'une intervention plus vigoureuse : « La violence est partout. Elle menace notre société [...]. Ceux qui croient qu'elle n'est ni plus forte, ni plus fréquente qu'elle n'était [...] font l'autruche. Pendant qu'ils cachent leur tête dans le sable, les plumes qui leur restent dans le dos s'envoleront, arrachées par le vent des contestations, et par le souffle des explosions" (P.9, Léauté, 1977).

Oracles des temps modernes, les sondages jugent les conséquences de cette apparente montée de violence par la peur qu'elle suscite dans l'opinion publique et en même temps prennent cette peur comme une mesure de la violence qui augmente. Autrement dit, l'augmentation apparente de la peur dans l'opinion publique est supposée refléter la réalité de la violence actuelle, révéler la vérité sur les faits. Pour prendre un exemple, le récent et fameux rapport Figgie (1980) sur la peur du crime aux États-Unis, indique que quatre Américains sur dix disent craindre d'être victimes d'un acte de violence et approximativement le même nombre affirme ne pas se sentir en sécurité lorsque seuls dans certains lieux, même familiers. Les conclusions des auteurs illustrent bien leurs visées pédagogiques sinon démagogiques : "Fear of crime is slowly paralyzing American Society [...] Americans have today become afraid of one another [...] " et d'ajouter, " telling the truth about how fear of crime has affected our lives may awaken the public to the urgent need to do something about it" (p. 2)1.

On peut se demander si, à force de parler de la menace que fait peser la violence et le crime sur la collectivité, on n'arrive pas à une situation paradoxale où le mythe devient presque plus réel que la " réalité " elle-même. Comme le soulignaient Weis et Milakovich (1974) : "Fear of crime, reports about it and the political misuses of that fear have become a problem as serious as crime itself" (p. 27).

* Chercheur agrégé au Centre international de criminologie comparée, Université de Montréal.

1. In : Criminal Justice Newsletter, vol. 11, n²., 1980. 
La peur, réaction naturelle de légitime défense face à un danger, n'est pas toujours employée à bon escient et peut devenir, nous dit Simenon lui-même, "un ennemi plus dangereux que tous les autres " (p. 32, 1967). Individuelle, la peur peut devenir cause de détresse, de blocage et de repli sur soi. Collective, elle peut encore conduire à des comportements aberrants de défense, à l'intolérance ou à l'hostilité. Il n'est qu'à survoler l'histoire, pour découvrir le nombre et l'importance des peurs qui ont entraîné des paniques, de la violence, ou ont conduit à des persécutions contre ceux désignés comme boucs émissaires. Sans vouloir entrer dans le jeu d'une pronostication de l'avenir, il est certain que la peur joue un rôle dans les réactions face à la déviance et à la criminalitéz 2 . Dans cette perspective, si la peur augmente, comme tentent de le montrer les sondages, il est nécessaire de regarder au-delà des chiffres cités pour voir ce que les gens signifient lorsqu'ils répondent à des questions sur leurs peurs.

\section{LA PEUR COMME " CONSTRUIT HYPOTHÉTIQUE "}

Bien que, depuis une dizaine d'années, on assiste à une prolifération d'études sur les réactions envers le crime, les mesures développées sont inconsistantes d'une recherche à l'autre et manquent souvent de rigueur sur le plan conceptuel (c.f. DuBow et al. 1979). Rien n'est plus difficile en effet à analyser que la peur qui reste éminemment subjective. La difficulté s'accroît lorsqu'il s'agit, non pas d'étudier la peur vécue par une personne face à un danger présent, mais comme reconstruction ou représentation. Ainsi, quand nous évoquons la peur actuelle de marcher seul dans la rue le soir, ou encore lorsque nous indiquons que le meurtre est ce que les gens redoutent le plus, nous renvoyons à une crainte dont l'origine réside dans l'expérience du sujet ou bien à une attitude qui sous-entend une certaine représentation du danger et de la menace. La peur est ici une réflexion sur sa propre expérience ou l'habitude que l'on a de redouter telle ou telle menace (liée à un danger réel ou imaginaire). Dans ce sens, on peut dire que l'expérience de peur observée ne peut être saisie directement mais seulement inférée à partir d'un ensemble d'indicateurs cognitifs (les idées et les images), affectifs (les sentiments, émotions...) et conatifs (les actions prises ou anticipées). Comme " construit " hypothétique, la peur pose donc les mêmes problèmes conceptuels et méthodologiques que toute

2. En France, qui n'est pourtant pas encore Chicago, des groupes d'auto-défense se sont constitués, de malheureux incidents se sont produits par peur, etc. 
notion non mesurable directement mais qui doit être inférée à partir d'un ensemble de réponses ${ }^{3}$.

Nous ne présenterons pas une évaluation des multiples indicateurs qui ont été utilisés jusqu'ici, une excellente revue critique de la littérature dans ce domaine ayant été faite récemment par DuBow et al. (1979). Nous voudrions plutôt, en nous aidant de nos propres travaux (Brillon, Louis Guérin et Lamarche, 1982), souligner certains aspects apparus comme particulièrement importants dans l'élaboration de mesures de peur et sur lesquels, peut-être, on n'a pas assez insisté.

\section{CONTENU DE LA PEUR : PEUR ABSTRAITE ET PEUR CONCRÈTE}

Un premier aspect qui nous semble essentiel à distinguer lorsqu'on mesure la peur du crime, est le degré de généralité auquel on se situe. Le crime, selon qu'il s'agit d'un phénomène général (abstrait et distant) se rapportant à la société ou à des lieux inhabituels, ou d'un phénomène local particulier (concret et proche) en rapport avec le vécu quotidien, constitue une réalité différente; et les représentations du danger à ces deux niveaux de généralité et de proximité, ne sont certainement pas les mêmes.

En effet l'impact de la criminalité apparaît quantitativement et qualitativement différent selon qu'il s'agit d'une menace concrète et immédiate dans l'environnement proche ou d'une menace plus ou moins abstraite et lointaine dans des endroits distants ou encore à un niveau plus général comme problème national. Dans l'ensemble, les gens ont toujours tendance à percevoir les taux de criminalité comme plus élevés et progressant plus rapidement ailleurs que dans leur propre localité. Également le crime et la délinquance sont jugés comme des problèmes graves et parmi les plus importants (parmi les trois problèmes les plus importants) sur le plan social alors que leur saillance reste très faible au niveau personnel. Dans la vie quotidienne, des problèmes tels que la santé, l'argent, le travail ou la famille etc., sont des préoccupations beaucoup plus courantes et importantes que la criminalité4. Enfin, ce sont les crimes les plus violents qui suscitent la plus grande peur mais, en même temps, dont l'incidence au niveau personnel est jugée comme la plus improbable.

3. Degré de généralité des mesures ; proximité et familiarité de l'objet d'attitude ; relation entre attitude et comportement réel ; unidimensionalité ; désirabilité sociale...

4. Le format de la question influence beaucoup les réponses. La place accordée à la criminalité, comme problème social ou personnel est toujours moins importante lorsque la question est ouverte et donc aucune réponse suggérée, que dans une question fermée incluant comme choix de réponse cette alternative. 
On voit ainsi se profiler, à travers ces exemples, un certain hiatus entre les représentations et la peur que peut engendrer la criminalité en général et celle concrètement vécue au niveau personnel en relation à la criminalité dans l'environnement immédiat et quotidien.

On peut expliquer en partie cette apparente contradiction par le type d'informations et d'expériences sur lesquelles se fondent les représentations et les peurs à ces deux niveaux.

\section{a) La peur abstraite du crime}

Les gens, pour se former une idée de la criminalité en dehors des lieux qu'ils fréquentent, ne peuvent le faire qu'à partir d'une expérience relativement abstraite, en s'appuyant sur des informations qui, en fait, sont constituées de fragments d'images, d'opinions entendues, se mêlant à des impressions personnelles. Les faits sont ici très secondaires. Autrement dit, les images participent davantage de l'idéologie que de la réalité, dans la mesure où il n'existe comme points de repère que des informations qui arrivent aux gens et sont retenues selon plusieurs mécanismes de sélection, simplification et généralisation, amenant à exagérer certains événements au profit d'autres. Le donné est reconstruit ici dans le contexte des valeurs et des notions normatives.

D'une manière générale, le sentiment que la violence augmente est profondément enraciné dans l'opinion publique. Ce sentiment se fonde sur les impressions les plus diverses selon les orientations profondes. Pour les uns, c'est la conséquence de l'éclatement des structures traditionnelles, l'excès de libéralisme dans le contrôle des comportements ou encore la dégradation des valeurs morales, le laxisme des juges, etc. Pour les autres, ce sont les inégalités sociales qui ne cessent de croître, la crise économique, le rythme effréné de la vie et l'urbanisation. Quelles que soient les explications, cette vision repose sur 'amalgame entre violence et criminalité. Mais ce sentiment résulte aussi du caractère même, à la fois marquant et effrayant, des crimes crapuleux ou spectaculaires, auxquels les media d'information donnent une résonnance particulière. Ces images, en nourrissant l'imaginaire social d'une réalité tronquée, fournissent des objets de peur, alimentent de références exemplaires le discours pour justifier la montée de la violence. Cette construction tronquée de la réalité dans le discours public est évidente. Ainsi, il y a des omissions frappantes dans ce discours de l'opinion publique, comme le montraient nos entretiens (Baril, 1977). En effet, pas un mot sur la violence domestique qui, pourtant, occupe une place importante dans l'ensemble de la violence. Mais la violence entre les membres d'une même famille a quelque 
chose d'inacceptable et, en même temps, remet en question l'image officielle, rassurante de la famille ou celle des rapports entre les sexes. Certaines féministes ont largementt épilogué sur ce silence. Pas un mot non plus sur la criminalité économique des cols blancs... Il est frappant de voir à ce niveau de généralité, que la représentation du crime et du danger est non seulement éminemment subjective et floue mais myope sélectivement. Les éléments retenus ne le sont pas au hasard. L'événement, toujours rapporté aux structures mêmes de l'organisation sociale et politique, devient ainsi l'expression de cette organisation, l'effet de l'ordre dominant. On voit ainsi que l'appréhension de la criminalité à ce niveau nous renseigne autant sur la portée sociale du phénomène que sur le degré de tolérance sociale. Le crime et la déviance sont en effet des objets sociaux privilégiés pour exprimer les modalités des rapports.

Prendre ces mesures comme des mesures de l'impact du crime dans la vie quotidienne des gens entretient certainement une confusion entre ce qu'on pourrait appeler la peur abstraite - qui reste une menace lointaine et très générale - et la peur concrète, exprimant une crainte de victimisation face à une menace dans l'environnement immédiat.

Ces deux types de peur se distinguent, non seulement par les informations et les expériences qui les fondent mais, également, par le fait qu'elles n'ont pas la même qualité d'imminence et qu'elles entraînent des réactions différentes.

Ainsi, on verra que la peur " abstraite "entraîne une réaction sociale pour des solutions collectives du problème criminel (contrôle, prévention, services aux victimes, mesures plus répressives). Ces solutions, préférées ou supportées dépendent des orientations profondes et des images associées du crime, des criminels et de la justice. La peur " concrète" conduit en revanche à des solutions individuelles immédiates pour minimiser les risques perçus (évitement, protection, assurance, recherche et échange d'information, participation à des programmes...); le choix pour l'une ou l'autre de ces solutions individuelles étant déterminé par les ressources personnelles, le cycle de vie, etc.

\section{b) La peur concrète du crime}

Lorsqu'on passe, par contre, à un terrain plus familier, la criminalité peut être éprouvée comme une réalité quotidienne et la perception des dangers s'appuie sur certains indices de l'environnement im- 
médiat (activités de la police, personnes ou situations suspectes...) et sur une expérience directe ou indirecte (comme témoin ou par personne interposée) de victimisation. Ces repères permettent une évaluation plus réaliste du problème criminel au niveau local, des taux et du changement ainsi que des dangers qui existent. Les perceptions, à ce niveau, que se font les gens de la criminalité environnante, de leurs risques de victimisation, apparaissent des réponses relativement réalistes et sensibles aux risques objectifs. Ainsi la saillance de la criminalité est plus importante dans les zones où le taux de criminalité officiel est plus élevé et dans les grandes villes où les gens sont plus exposés en comparaison des zones rurales. La saillance est également plus élevée, parmi les personnes objectivement plus vulnérables (faible revenu, personnes seules, locataires...) ou déjà victimes d'actes criminels.

Cependant, à l'intérieur de ces zones de résidence et parmi certains groupes de personnes, notamment les femmes et les personnes âgées apparemment moins victimisées, on constate une plus grande peur du crime. Devant cette relation inverse entre le taux réel de victimisation et la peur, certains ont voulu conclure au caractère irrationnal de cette peur. Pourtant ici, en se limitant à la probabilité de victimisation réelle ou perçue, on néglige certains aspects qualificatifs de la criminalité qui peuvent être tout autant, sinon plus effrayants pour certaines personnes. Ainsi, la capacité de se défendre, la gravité des conséquences et la possibilité de récupération lors d'une victimisation sont certainement des facteurs importants, dans la peur. Une personne âgée a généralement moins de ressources physiques pour se défendre et constitue une cible plus facile pour les criminels. Les conséquences d'une agression peuvent être plus graves, même un vol de quelques dollars peut avoir des effets dramatiques dans certains cas. De la même façon, si cette personne tombe lors d'une altercation, les conséquences peuvent être plus sérieuses que pour une personne plus jeune, sans parler des conséquences psychiques.

Également, le type de crime dont on a le plus peur est important à saisir. En effet, la peur d'être violée ou attaquée physiquement est qualitativement différente de celle d'être cambriolée ou que l'on vole sa voiture ou encore son sac. On a montré ainsi que la peur élevée des femmes en général, était moins la conséquence de traits de personnalité que le type de crimes dont elles étaient victimes, notamment le viol (S. Riger, 1978). Enfin l'intégration dans le quartier joue également un rôle important. Ainsi, la connaissance des voisins, la familiarité avec les lieux, facilitent dans une certaine mesure le contrôle de la situation criminelle. Elles permettent plus aisément de repérer des inconnus ou 
des situations suspectes; d'éviter certains endroits jugés comme dangereux ; de savoir où trouver de l'aide en cas de problème... Dans ce sens l'intégration augmente le sentiment de contrôle sur la situation criminelle et diminue celui de vulnérabilité.

On voit ainsi que pour comprendre la peur concrète du crime, il ne s'agit pas seulement de se focaliser sur l'aspect quantitatif de l'impact du crime (degré d'exposition et risques objectifs) mais également de tenir compte de l'aspect qualitatif lié à l'impact différent du crime et au sentiment de vulnérabilité (capacité de se défendre, type de crimes saillants, gravité des conséquences et possibilité de récupération).

La peur concrète, à la différence de la peur abstraite, semble ainsi refléter de façon beaucoup plus réaliste l'impact réel de la criminalité dans la vie quotidienne des gens. Elle apparaît comme une réponse relativement adaptée aux risques et à la vulnérabilité. Plus la sjtuation est non familière, plus les gens auront tendance à utiliser des définitions impersonnelles, normatives et stéréotypées. À l'inverse, une situation familière induira des définitions moins contraintes par des normes externes et d'autant plus personnelles qu'elles se basent sur des expériences singulières. Ainsi, on a pu voir que les informations véhiculées par les médias ont moins d'effets sur les gens résidant dans des zones fortement criminogènes, dans la mesure où ils reçoivent plus d'informations directes sur la criminalité de leur environnement immédiat que ceux de zones faiblement criminogènes.

Quels que soient les aspects du phénomène étudié, on retrouve ce décalage entre ce qui est déterminé par des informations et une expérience directe et ce qui, faute d'autres points de repère, se nourrit de croyances et d'une imagerie populaire. Si l'on prend, par exemple, les jugements qui sont portés sur les différentes agences du système, la plus grande proximité de la police avec les citoyens rend leurs activités plus visibles que celles des autres agences. Ceci permet aux gens d'évaluer la police dans leur quartier à partir d'indices concrets (sécurité du quartier, fréquence des patrouilles...). En revanche, le peu de contacts et la faible visibilité des actions des tribunaux ou des services correctionnels, ne permettent de se faire une idée qu'à travers les médias d'information, et laissent la place à toute l'imagerie populaire qui circule sur les juges, les prisons, les détenus... C'est ici le criminel violent, récidiviste, irrécupérable qui sert d'étalon pour juger de la gravité du problème, de l'efficacité du système de justice et de la sévérité des tribunaux et des juges ${ }^{5}$. La police seule échappant en partie à ce méca-

5. Ceci invite à beaucoup de prudence lorsqu'on affirme que les gens sont punitifs, 
nisme globalisant, on comprend mieux les évaluations positives qui sont faites de leurs activités, la plupart des gens se sentant en sécurité dans leur quartier (peur concrète). Par contre, l'inquiétude générale (peur abstraite) devant la montée de la violence et l'image alarmiste, qui existe à ce niveau de généralisation, ne peuvent qu"induire un jugement négatif ${ }^{6}$ sur les activités de ces autres agences.

On voit ainsi que certaines contradictions apparentes s'expliquent lorsqu'on distingue ces deux types de peur abstraite et concrète et les connaissances expérientielles qui les fondent. La première apparaît davantage exprimer une préoccupation générale et un jugement de valeurs sur l'importance sociale du problème criminel. Elle mesure plus particulièrement le seuil de tolérance sociale et la punitivité et se fonde sur des idées, des croyances qui n'ont que peu de relation avec les faits. La seconde, en revanche, est une réponse relativement adaptée à une menace directe, perçue dans l'environnement immédiat et prend appui sur des indices concrets. Elle constitue la réelle mesure de l'impact actuel de la criminalité dans la vie quotidienne des gens. Ces définitions impliquent que l'on doit développer des mesures différentes pour ces deux types de peur. La peur "concrète" doit être analysée à partir des variables contextuelles et individuelles qui différencient la population selon l'exposition, les risques et le sentiment de vulnérabilité, selon les ressources dont chacun dispose pour se défendre face à une menace de victimisation et selon les conséquences réelles ou appréhendées d'une victimisation. En revanche, pour la peur "abstraite ", les variables fondamentales sont les idées et les images que les gens se font de la criminalité, des criminels et de la justice ; et, au-delà, leur vision du monde et de la société qui produit un type de croyances ou un autre.

\section{FORME DE LA PEUR : PEUR SPONTANÉE ET PEUR RÉFLÉCHIE}

Ces précisions sur le contenu de la peur selon la proximité et la généralité du danger, laissent encore dans l'ombre une autre ambiguïté fondamentale pour comprendre la signification des réponses de peur : la forme de la peur que l'on peut observer. En effet le contenu de la peur porte sur l'objet, ce qui fait peur et ce qui incite à avoir peur. La forme de la peur représente les modalités d'expression de la peur,

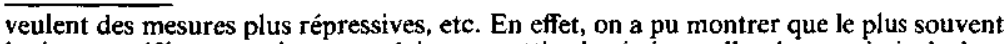
les images référentes qui sous-tendaient ces attitudes étaient celles de ces criminels dangereux.

6. Jugées comme trop laxistes ou inadaptées selon les orientations idéologiques. 
comme réaction plus ou moins élaborée et réfléchie devant le danger, indépendamment de la représentation de ce danger.

Nous sommes ici au point de départ d'un autre malentendu qui s'attache aux mesures de la peur. On n'a guère distingué ce qu'on pourrait appeler la peur "spontanée " qui est une réaction automatique et non réfléchie devant le danger en général et la peur " réfléchie" résultant d'un jugement raisonné sur les menaces concrètes. Si la première s'exprime en l'absence d'objets déterminés, la seconde en revanche s'appuie sur des éléments concrets de la situation et réfléchit dans une certaine mesure les risques perçus (réels ou imaginaires).

a) Les processus primaires et secondaires dans la peur

Un des apports de la psychanalyse a été de distinguer les processus primaires et les processus secondaires dans le mode de fonctionnement du psychisme au niveau des représentations. Les représentations primaires se font sur un mode hallucinatoire et se caractérisent par leur aspect impulsif et leur intensité, mettant en cuvre des mécanismes de déplacement, de condensation et de surdétermination. Autrement dit, une représentation souvent d'apparence insignifiante peut se voir attribuer toute la valeur psychique, la signification, l'intensité originellement atiribuées à une autre. D'autre part en une représentation unique peuvent confluer toutes les significations portées par les chaînes associatives qui viennent s'y croiser. Enfin la représentation renvoie à une pluralité de facteurs déterminants et donc est constituée de glissements et de superpositions de sens, elle n'est jamais le signe univoque d'un contenu unique.

On voit déjà ici la difficulté pour interpréter de telles représentations. La peur du danger à ce niveau peut donc bien contenir ce qu'elle veut et autant qu'elle veut. $\dot{A}$ partir du moment où les choses n'ont d'autre sens que celui qu'on leur impose, comment ne pas voir dans leur visage le reflet de notre propre visage ? C'est ainsi que la peur spontanée - fantasme né dans notre esprit - commence à délirer et nous avec elle.

Il ne s'agit pas de dire que la peur à ce niveau, n'a pas de réalité mais seulement qu'elle n'est pas réelle. Autrement dit, la peur est la peur de la peur (génitif subjectif, génitif objectif), elle est à elle-même son propre fondement et sa fin, elle est cause d'elle-même.

Cependant, il nous faut sortir de ces fantasmes pour attribuer à un objet une existence. Nous nous interrogeons maintenant sur la nature de cette effraction. Ici encore, en effet, un décalage se produit. Le 
décalage continue à être déterminé par l'expérience et les informations mais également par la capacité d'élaboration pour donner un sens à nos peurs. C'est là qu'intervient la notion de représentations secondaires.

En opposition aux réponses automatiques, spontanées êt non réfléchies qui fonctionnent sur le mode fantasmatique et ont une apparence d'incohérence, les représentations secondaires se présentent sous la forme d'un scénario relativement cohérent et compréhensible. Elles sont soumises au principe de réalité par la médiation du système perceptif et établissent des voies associatives qui prennent en considération l'intelligibilité. Elles constituent, comme leur nom l'indique, un deuxième temps du travail d'expression des représentations. Ces représentations secondaires seront surtout à l'œuvre lorsqu'il y a un effort de réflexion et à fortiori quand il y a un discours sur le crime et le danger qu'il représente. La notion d'élaboration fournirait ainsi une charnière entre la peur "spontanée " et la peur "réfléchie " et expliquerait leur forme différente.

b) La peur spontanée du crime

À un premier niveau non réfléchi et automatique, lorsque les gens pensent au crime, au criminel, les premières images qui surgissent spontanément sont des images d'une forte intensité et dominées par des intérêts immédiats. La caractéristique de ces images spontanées sont leur nature a-topique et a-temporelle, elles circulent sans repérage aucun et de ce fait n'ont plus aucune mesure avec l'événement. Autrement dit, l'identification, qui est le principe de reconnaissance, n'est pas efficace ici.

Un discours qui raconte le déroulement d'une conduite criminelle, avec ses causes et ses effets, oblige par son travail même de rationalisation à une reconstruction proche de la réalité. Les explications obligent à établir des relations causales pour définir l'acte, sa finalité. Au contraire, à ce niveau spontané d'expression, ces images circulent dans une destructuration même de la relation de causalité, et sont des fragments qui participent à une autre logique, celle du mythe populaire qui se nourrit des crimes sanglants ou crapuleux, du spectaculaire, des cas particuliers et atypiques qui excitent l'imagination. L'obsession de la violence multiplie les hallucinations du danger et ce danger est toujours sursignifié englobant d'autres menaces, d'autres inquiétudes : le chômage, la maladie, etc. 
Ce niveau des perceptions, semble relativement indépendant de l'idéologie et des orientations profondes mais puise ses idées et ses images dans les journaux, la télévivion, etc. Les mass-media pénétrant dans chaque foyer dirigent les images et les uniformisent; et par une espèce de contamination sociale, les mêmes idées et les mêmes images sont évoquées de façon répétitive, se propagent. De sorte qu'elles sont constamment saillantes et, dans la conscience, immédiatement disponibles à la moindre stimulation. En effet, la saillance de ces images se reconnaît avec la rapidité avec laquelle elles émergent dans la conscience. Très pauvres et peu nuancées au niveau cognitif, elles sont en revanche émotivement très chargées, suscitant diverses réactions de peur, de répulsion, d'exaspération, d'agressivité, de fascination, etc. Elles ont ainsi une qualité impérative et irrésistible qui manque aux pensées plus élaborées et procèdent par simplification, assimilation et généralisation. Ce sont à ces images fortes que l'on a recours spontanément et à un niveau non réfléchi pour juger de la situation criminelle, comme si le crime ne pouvait signifier que violence. Des nombreuses images que l'on peut avoir, on ne retient ici qu'une seule qui, par déplacements, condensations, subsume et vient effacer l'hétérogénéité de son surgissement. L'événement vu ou entendu a un effet de grossissement et devient le tenseur dont elle a besoin pour être relancée. Cet événement polarise et actualise la peur spontanée de la violence mais son intensité n'est pas repérable sur lui, elle s'inscrit dans l'accumulation même d'images qui sont simultanément effectuées. L'intéressant est de retenir ici avec quelles très petites pièces d'information, de tels effets de canalisation des influx émotionnels peuvent être obtenus : l'événement singulier devient toute la réalité criminelle et la seule, alors qu'il suffit d'une minute de réflexion pour s'apercevoir que la réalité criminelle ne peut se réduire à cette seule réalité. Mais déjà on passe ici à un autre niveau d'expression.

\section{c) La peur réfléchie du crime}

Chacun peut faire l'expérience de la prégnance de telles images intenses et globalisantes devant un événement violent ou dans l'obscurité d'un lieu mal famé ou encore à un niveau non réfléchi lorsqu'il pense spontanément au crime... Cependant, il existe un vaste réseau imagé par ailleurs. Si, d'un côté, le crime se présente sous ses aspects les plus violents et irrationnels, de l'autre côté, il nous devient plus familier, incarné dans des personnes qui agissent suivant les circonstances auxquelles se rapporte l'interprétation et l'on passe des cas extrêmes à d'autres plus quotidiens. 
Comment s'opère ce passage entre la représentation imaginaire et globalisante du crime et la représentation d'une réalité plus quotidienne, nuancée et moins effrayante? "S'imaginer, comme dit Casamayor, qu'on est entouré de violence, de bandits dangereux et de brutes sadiques est aussi paralysant que de croire qu'il y a du poison dans tous les plats. Au bout c'est la folie par inquiétude" (p. 90, 1975). À un second palier, on débouche au niveau de la réflexion que chaque individu, chaque groupe ou société opère sur ses propres peurs pour les exorciser en partie. Ce n'est que lorsqu'un discours peut s'élaborer, qu'il est possible d'effectuer une distanciation à l'égard de l'emprise subjugante des images spontanées de peur.

La réflexion permet le recul nécessaire, les idées et les images sur le crime deviennent perméables au raisonnement, au doute, à l'évidence des faits contraires. Le crime est personnifié par le visage, les traits supposés de certains criminels et les détails de leur vie et de leur(s) crime(s). C'est en s'appuyant sur ces exemples types que la plupart des gens vont interpréter le phénomène criminel et expliquer la conduite des criminels, leurs caractéristiques, leurs motifs et leur dangerosité. Pour expliquer la possibilité du passage à l'acte, on va chercher à comprendre la chaîne des déterminismes qui conduit une personne à commettre un crime. Dès qu'un lien est établi, le champ s'élargit considérablement et permet d'exprimer toute une gamme de comportements et aussi toute une conception du problème criminel : Qui est criminel ? pourquoi devient-on criminel ? peut-on changer et comment?

Les théories profanes sur la criminalité retrouvent ou restituent les théories "scientifiques" et sont tout aussi nuancées et diversifiées. À travers les exemples cités, les comportements et les individus expliqués, les situations définies, on voit se profiler tout un système définissant et classant les bons des mauvais, les petits criminels des vrais criminels et les malades; ceux qui doivent être punis sévèrement ou écartés et ceux que l'on peut tolérer ou chercher à réinsérer dans la société. Le raisonnement oblige à être nuancé, à se rapporter à une réalité sociale concrète où peuvent s'établir des liens de responsabilité ou d'identification vis-à-vis des criminels dont il est question. On se sent concerné, on est convainccu qu'on punit justement et à bon escient.

Ces théories du sens commun ou profanes révèlent des préjugés, des distorsions et des simplifications qui ne sont jamais neutres mais correspondent à une vision du monde. Les intérêts sociaux transparaissent à travers les images, les informations et les définitions que chaque individu ou groupe se fait et se donne du crime, des criminels 
et de la justice. Se représenter ce n'est pas seulement sélectionner certains éléments en en délaissant d'autres, mais c'est aussi élaborer un système d'interprétation qui permet de déceler, prédire ou préparer son action ou réaction. Qualifier, par exemple, une personne de vrai criminel permet de catégoriser ses gestes ou paroles, de justifier ses propres réactions à son égard; et même davantage de voir dans cette personne les effets d'une situation sociale ou d'un mécanisme psychologique, de reconstituer les divers scénarios de ses rapports avec son milieu, ses parents, etc., comme si on en avait été témoin.

Si la peur spontanée ne différencie pas et est rapportée à une seule image, globalisante, qui envahit tout le champ de conscience, où se confond le réel et la fiction, la peur réfléchie en revanche oriente vers le bien fondé de certains dangers qui sont clairement identifiés, nommés et circonscrits. Ces images de danger s'enchaînent avec celles des moyens de défense pour réduire la menace ou minimiser les risques. Alors que la peur spontanée, coupée d'objets identifiables, reste désorientée.

Deux remarques pour finir. La première concerne le rôle que l'on peut attribuer à ces différents contenus (abstrait et concret) et formes d'expression (spontanée et réfléchie) de la peur ? Jusqu'ici, ils n'ont guère été systématiquement distingués et il est donc difficile d'en connaître la portée. Également la jonction entre le degré de généralité et le niveau d'expressivité de la peur, risque de rester énigmatique dans l'étude qu'on vient de faire, faute des éclaircissements nécessaires. Il s'agissait surtout ici d'ouvrir des voies, de tenter d'élucider certaines confusions et ambiguïtés que nos résultats semblaient suggérer. Il faudrait une longue étude plus élaborée pour rendre celle ébauchée plus systématique et d'une autre portée.

La deuxième observation est plus concrète et immédiate, si j'ose dire après cette discussion ! La peur du crime, comme on l'a montrée, se laisse difficilement enfermer dans une seule définition et l'équivoque sur sa signification permet de mesurer tout et n'importe quoi si l'on ne spécifie pas ce que l'on mesure. La "réalité " de la peur semble fort différente si l'on mesure la peur abstraite qui reflète une plus ou moins grande tolérance sociale face au problème criminel et la peur concrète basée sur des risques objectifs et le sentiment de vulnérabilité. La première dépend des orientations profondes et les faits restent ici secondaires. La deuxième prend appui sur des indices concrets de l'environnement immédiat et l'incidence réelle de la criminalité dans la vie quotidienne. De la même façon à un premier niveau non réfléchi, on a une réponse spontanée de peur face à une criminalité dont l'image do- 
minante est celle de violence et d'horreur. Ce sont ces images automatiques et très intenses que semblent recueillir le plus souvent les sondages d'opinion. En effet, les gens doivent répondre à des questions utilisant des termes fortement chargés : crime, criminel, prison, etc., qui évoquent immédiatement des images de violence. D'autre part ces questions auxquelles les gens doivent réagir rapidement favorisent l'émergeance des images spontanées et non réfléchies, celles qui surgissent le plus facilement et le plus fréquemment. Enfin, comme on l'a longuement discuté, ce n'est que dans le discours que peut s'élaborer une réflexion et seule la narration peut accomplir une fonction de liaison et de désintensification, en même temps qu'elle ouvre le champ de représentation. À ce niveau d'expression, la peur réfléchit la situation criminelle d'une façon beaucoup plus nuancée qui, en fait, est aussi plus proche de ce qui est vécu dans la quotidienneté.

On ne peut s'étonner alors, devant les résultats des sondages qui constatent une peur étendue parmi le public face au crime et une apparente intolérance vis-à-vis des criminels. Les évaluations qui sont faites du phénomène criminel, du danger ainsi que de l'efficacité de la justice recourent le plus souvent à cette peur abstraite et spontanée.

Le degré de généralité (abstrait-concret) auquel se situent les différentes mesures élaborées et le niveau d'expressivité (spontanéréfléchi) qu'elles permettent de saisir, restent encore des problèmes mal élucidés. Un effort conceptuel et méthodologique permettrait certainement d'éviter des confusions et des ambiguïtés dans les résultats et plus important encore, de départager le mythe de la réalité.

\section{BIBLIOGRAPHIE}

BARIL, M. (1977) : L'Image de la violence au Québec, rapport $n^{\circ} 4$, Manuscrit inédit, C.I.C.C., G.R.A.C., Université de Montréal.

BRILLON, Y., LOUIS-GUÉRIN, C., LAMARCHE, M.C. (1982) : Les attitudes du public canadien envers les politiques criminelles. Manuscrit inédit, C.I.C.C., G.R.A.C., Université de Montréal.

CASAMAYOR (1975) : La tolérance, Paris, Gallimard.

Criminal Justice Newsletter (1980) : "Special Issue on the Public's Fear of Crime ", vol. $11, n^{2} 22$, p. 1-5.

DUBOW et al (1979) : Reactions to Crime : a Critical Review of the Litterature, Center for Urban Affairs, Northwestern Univ., 1979.

LEAUTÉ, J. (1977) : Notre violence, Paris, Denoël. Réponses à la violence, Rapport du comité đ’étude sur la violence, la criminalité et la délinquence, présidé par A. Peyrefitte, Paris, Presses Pocket, 1977.

RIGER, S. et al (1978) : "Women's Fear of Crime : from Blaming to Restricting the Victim ", Victimology 3 (3 et 4), p. 274-284.

SIMENON, G. (1967) : Oeuvres complètes, Paris, Gallimard. 
WEIS, K., MILAKOVICH, M.E. (1974) : "Political Misuses of Crime Rates ", Society, vol. $11, n^{\circ} 5$, p. $22-23$. 